\title{
Silyl Functionalization of Polyolefins
}

\author{
Bhanu P. S. Chauhan* and Bharathi Balagam
}

Nanomaterials Laboratory of Center for Engineered Polymeric Materials (CePM), Department of Chemistry and Graduate Center, City University of New York at College of Staten Island, 2800 Victory Blvd., Staten Island, New York 10314.

\section{General Information}

All of the experiments and manipulations were performed under dry oxygen-free nitrogen using standard Schlenk-line techniques. All the Solvents were purchased from EM science (Merck) and distilled over sodium/benzophenone before use. PMHS (Mw $\approx$ 2000), $\mathrm{Me}_{2} \mathrm{Pt}(\mathrm{COD}), 1,2$-polybutadiene (Mn 2200) and silanes were purchased from Aldrich Chemical Co., and Gelest Chemical Co. and used without further purification. ${ }^{1} \mathrm{H}$ NMR, ${ }^{13} \mathrm{C}$ NMR and ${ }^{29} \mathrm{Si}$ NMR spectra were recorded on $200 \mathrm{MHz}$ and $600 \mathrm{MHz}$ Varian Unity NMR instruments with $\mathrm{CDCl}_{3}$ as an internal standard. GPC analysis was carried out on Alliance GPCV 200 (Water) instrument, equipped with two silica columns, HRSE and HR-1 with the pore size range of $100-5000$ and $2000-4 \times 10^{6}$ respectively. This instrument was calibrated using polystyrene standards. THF was used as an eluent at the flow rate of $1 \mathrm{ml} / \mathrm{min}$ at $40{ }^{0} \mathrm{C}$. A third order calibration curve was used to measure the molecular weight of unknown samples. Philips CM 100 transmission electron microscope (TEM) was employed to examine the reaction mixture for the presence of Ptnanoclusters. Scanning electron microscope Amray 1910 (SEM) was used to analyze solid Pt-nanocluster. The amount of Pt present in the cluster was determined on the basis of SEM data, which is $~ 0.001 \mathrm{mmol}$ per $0.01 \mathrm{~g}$ of the solid.

\section{Synthesis of Pt-nanocluster Catalyst}

$\mathrm{Me}_{2} \mathrm{Pt}$ (COD) (cod: 1,5 cyclo-octadiene) (0.25 mmol, $0.084 \mathrm{~g}$ ), and PMHS (10.00 mmol, $0.60 \mathrm{~mL}$ ) were added in $50 \mathrm{~mL}$ toluene in a 200-mL RB. Reaction mixture was stirred at $80{ }^{0} \mathrm{C}$ for $24 \mathrm{~h}$ under positive pressure of nitrogen. Reduction of $\mathrm{Me}_{2} \mathrm{Pt}$ (COD) was monitored with UV-vis spectroscopy, which revealed a gradual disappearance of peaks associated with the $\mathrm{Me}_{2} \mathrm{Pt}$ (COD) leading to a featureless spectrum. After verifying the presence of "Pt"-nanoclusters in the solution with EM-spectroscopy, flow of nitrogen was stopped and the flask was exposed to air. Further stirring of the solution in air 
yielded a black gummy solid. The solid was washed thoroughly with toluene/benzene $(100 \mathrm{~mL})$ to remove organic moieties. Air-drying of the gummy solid furnished a black powder, which was characterized with various spectroscopic techniques and used as hydrosilylation catalyst. ${ }^{29} \mathrm{Si}$ CP/MAS $\delta$-36.15 (D, Si-H), -81.46(T), -102.58(Q, $\left.\mathrm{SiO}_{2}\right)$, 112.26(Q, $\left.\mathrm{SiO}_{2}\right) .{ }^{13} \mathrm{C} \mathrm{CP} / \mathrm{MAS} \delta-3.35\left(-\mathrm{SiCH}_{3}\right)$. FT-IR spectra $\left(\mathrm{KBr}, \mathrm{cm}^{-1}\right) 2175(\mathrm{Si}-\mathrm{H})$, 1120 (Si-O-R), 1050 (Si-O-Si), 893 (Si-OH).

\section{General Procedure For Hydrosilylation of Polybutadienes}

A Schlenk tube $(10 \mathrm{~mL})$, equipped with magnetic stirrer and oil bath was charged with Pt-nanoclusters $(0.01 \mathrm{~g}, 0.001 \mathrm{mmol} \mathrm{Pt})$, degassed and flushed with dry Nitrogen. The PBD-1 (0.054g, 1mmol) dissolved in freshly prepared dry benzene $(2 \mathrm{~mL})$ was added to the Schlenk, followed by the addition of $\mathbf{2 a}(0.13 \mathrm{ml}, 1.2 \mathrm{mmol})$ under the constant flow of nitrogen. After few minutes of stirring, the reaction mixture turned into light brown homogeneous solution, indicating the formation of soluble nanoclusters. ${ }^{12}$ The reaction progress was monitored by ${ }^{1} \mathrm{H}$ NMR spectroscopy. On completion of the reaction, the solid catalyst was separated by centrifugation. The filtrate was evacuated to obtain the crude product. The product thus obtained was passed through the silica gel column (hexane) and analyzed by using GPC and ${ }^{1} \mathrm{H},{ }^{13} \mathrm{C}$, DEPT, ${ }^{29} \mathrm{Si}$ NMR techniques.

\section{Detailed NMR Analysis of the Product(s)}

\section{Polymer, 3a}

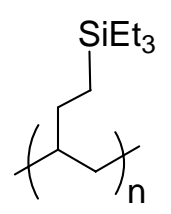

${ }^{1}$ HNMR (CDCl, $\left.200 \mathrm{MHz}\right): \delta$ (ppm) 0.44 (t, 9H), 0.85 (q, 6H), 0.0-2.0 (br, 7H).

${ }^{13}$ CNMR/DEPT $\left(\mathrm{CDCl}_{3}, 200 \mathrm{MHz}\right): \delta(\mathrm{ppm}) 3.29\left(-\mathrm{SiCH}_{2} \mathrm{CH}_{3}\right), 7.52\left(-\mathrm{SiCH}_{2} \mathrm{CH}_{3}\right)$, $27.0\left(-\mathrm{CH}_{2} \mathrm{CH}_{2} \mathrm{Si}\right), 34.7\left(-\mathrm{CHCH}_{2}-\right)$, $38.1\left(-\mathrm{CHCH}_{2}-\right)$.

${ }^{29} \mathrm{SiNMR}\left(\mathrm{CDCl}_{3}, 600 \mathrm{MHz}\right): \delta$ (ppm) 7.76.

\section{Polymer, 3b}




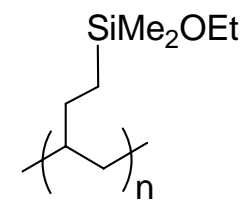

${ }^{1}$ HNMR (CDCl, $\left.200 \mathrm{MHz}\right): \delta$ (ppm) -0.04 (s, 6H), 0.2-2.0 (br, 7H), 1.05 (t, 3H), 3.52 (q, 2H).

${ }^{13}$ CNMR/DEPT $\left(\mathrm{CDCl}_{3}, 200 \mathrm{MHz}\right): \delta(\mathrm{ppm})-2.14\left(-\mathrm{SiCH}_{3}\right), 11.29\left(-\mathrm{CH}_{2} \mathrm{CH}_{2} \mathrm{Si}-\right)$, 18.305(- $\left.\mathrm{OCH}_{2} \mathrm{CH}_{3}\right), 25.79\left(-\mathrm{SiCH}_{2} \mathrm{CH}_{2}-\right)$, 33.57(- $\left.\mathrm{CHCH}_{2}-\right)$, $37.68\left(-\mathrm{CHCH}_{2}-\right), 58.16(-$ $\left.\mathrm{OCH}_{2} \mathrm{CH}_{3}\right)$.

${ }^{29} \mathrm{SiNMR}\left(\mathrm{CDCl}_{3}, 600 \mathrm{MHz}\right): \delta(\mathrm{ppm}) 18.01$

Polymer, 3c

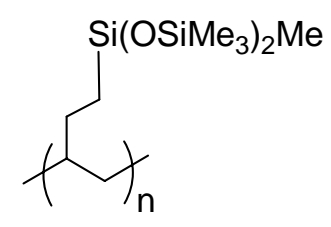

${ }^{1}$ HNMR (CDCl $\left.3,200 \mathrm{MHz}\right): \delta$ (ppm) -0.10 (s, 3H), -0.02 (s, 6H), 0.0-2.0 (br, 7H)

${ }^{13}$ CNMR/DEPT $\quad\left(\mathrm{CDCl}_{3}, \quad 200 \mathrm{MHz}\right): \quad \delta(\mathrm{ppm}) \quad-0.72 \quad\left(-\mathrm{SiCH}_{3} \mathrm{OSiCH}_{3}\right), \quad 1.62 \quad(-$ $\left.\mathrm{SiCH}_{3} \mathrm{OSiCH}_{3}\right), 12.93\left(-\mathrm{CH}_{2} \mathrm{CH}_{2} \mathrm{Si}-\right), 25.95\left(-\mathrm{CH}_{2} \mathrm{CH}_{2} \mathrm{Si}-\right), 33.7\left(-\mathrm{CHCH}_{2}-\right), 37.96$ ($\left.\mathrm{CHCH}_{2-}\right)$.

${ }^{29} \operatorname{SiNMR}\left(\mathrm{CDCl}_{3}, 600 \mathrm{MHz}\right): \delta(\mathrm{ppm})-20.36,7.02$.

\section{Polymer, 3d}

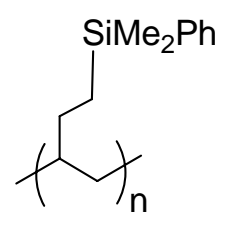

${ }^{1}$ HNMR (CDCl, $\left.200 \mathrm{MHz}\right): \delta$ (ppm) 0.27 (s, 3H), 0.4-2.0 (br, 7H), 7.34 (m, 3H), 7.50 (m, 2H).

${ }^{13}$ CNMR/DEPT $\left(\mathrm{CDCl}_{3}, 200 \mathrm{MHz}\right): \delta(\mathrm{ppm})-3.22\left(-\mathrm{SiCH}_{3} \mathrm{Ph}\right), 10.76\left(-\mathrm{CH}_{2} \mathrm{CH}_{2} \mathrm{Si}-\right)$, 26.72(-SiCH $\mathrm{CH}_{2}$ ), 33.7(- $\left.\mathrm{CHCH}_{2}-\right)$, $37.85\left(-\mathrm{CHCH}_{2}-\right), 127.49$ (-SiPh), $128.12(-\mathrm{SiPh})$, $128.54(-\mathrm{SiPh}), 133.3(-\mathrm{SiPh})$.

${ }^{29} \mathrm{SiNMR}\left(\mathrm{CDCl}_{3}, 600 \mathrm{MHz}\right): \delta(\mathrm{ppm})-1.795$

\section{Polymer, 3e}




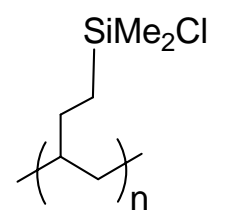

${ }^{1}$ HNMR $\left(\mathrm{CDCl}_{3}, 200 \mathrm{MHz}\right): \delta$ (ppm) 0.07 (s, 6H), 0.0-2 (br, 7H)

${ }^{13}$ CNMR/DEPT $\left(\mathrm{CDCl}_{3}, 200 \mathrm{MHz}\right): \delta(\mathrm{ppm}) 1.62\left(-\mathrm{SiCH}_{3}\right), 14.37\left(-\mathrm{CH}_{2} \mathrm{CH}_{2} \mathrm{Si}-\right)$, 26.1($\left.\mathrm{CH}_{2} \mathrm{CH}_{2} \mathrm{Si}-\right)$, $33.6\left(-\mathrm{CHCH}_{2}-\right)$, 38.1 (- $\left.\mathrm{CHCH}_{2}-\right)$.

${ }^{29} \mathrm{SiNMR}\left(\mathrm{CDCl}_{3}, 600 \mathrm{MHz}\right): \delta(\mathrm{ppm}) 32.97$.

Polymer, 3f

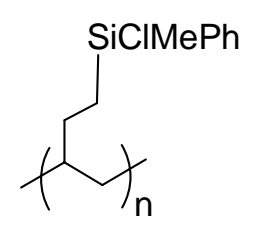

${ }^{1}$ HNMR (CDCl, $\left.200 \mathrm{MHz}\right): \delta$ (ppm) 0.49 (s, 3H), 0.0-2.0 (br, 7H), 7.23 (m, 3H), 7.45 (m, 2H).

${ }^{13}$ CNMR/DEPT (CDCl $\left.3,200 \mathrm{MHz}\right): \delta$ (ppm) $0.26\left(-\mathrm{SiCH}_{3}\right), 13.52\left(-\mathrm{CH}_{2} \mathrm{CH}_{2} \mathrm{Si}-\right), 25.83$ (- $\left.\mathrm{CH}_{2} \mathrm{CH}_{2} \mathrm{Si}-\right), 33.43\left(-\mathrm{CHCH}_{2}-\right), 37.86\left(-\mathrm{CHCH}_{2}-\right), 128.06$ (-SiPh), 130.27 (-SiPh), $133.27(-\mathrm{SiPh}), 135.24(-\mathrm{SiPh})$.

${ }^{29} \mathrm{SiNMR}\left(\mathrm{CDCl}_{3}, 600 \mathrm{MHz}\right): \delta$ (ppm) 22.17.

\section{Polymer, 3g}

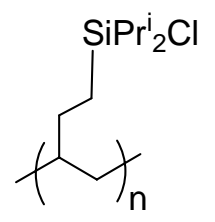

${ }^{1}$ HNMR (CDCl, $200 \mathrm{MHz}$ ): $\delta$ (ppm) 0.0-2 ( br, 21H)

${ }^{13}$ CNMR/DEPT ( $\left.\mathrm{CDCl}_{3}, 200 \mathrm{MHz}\right): \delta(\mathrm{ppm}) 8.05\left(-\mathrm{CH}_{2} \mathrm{CH}_{2} \mathrm{Si}-\right), 13.73\left(-\mathrm{SiCH}\left(\mathrm{CH}_{3}\right)_{2}\right)$, $16.99\left(-\mathrm{SiCH}\left(\mathrm{CH}_{3}\right)_{2}\right), 25.8\left(-\mathrm{CH}_{2} \mathrm{CH}_{2} \mathrm{Si}-\right), 33.93\left(-\mathrm{CHCH}_{2}-\right), 37.61\left(-\mathrm{CHCH}_{2}-\right)$.

${ }^{29} \mathrm{SiNMR}\left(\mathrm{CDCl}_{3}, 600 \mathrm{MHz}\right): \delta(\mathrm{ppm}) 36.8$.

\section{Polymer, 3h}

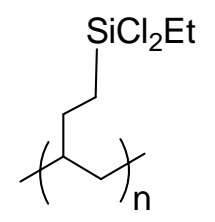

${ }^{1}$ HNMR (CDCl $3,600 \mathrm{MHz}$ ): $\delta$ (ppm) 0.0-2 (br, $12 \mathrm{H}$ )

${ }^{13}$ CNMR/DEPT (CDCl, $\left.200 \mathrm{MHz}\right): \delta(\mathrm{ppm}) 6.16\left(-\mathrm{SiCH}_{2} \mathrm{CH}_{3}\right), 12.52\left(-\mathrm{SiCH}_{2} \mathrm{CH}_{3}\right)$, $15.52\left(-\mathrm{CH}_{2} \mathrm{CH}_{2} \mathrm{Si}-\right), 25.37\left(-\mathrm{CH}_{2} \mathrm{CH}_{2} \mathrm{Si}-\right), 33.0\left(-\mathrm{CHCH}_{2}-\right), 37.7\left(-\mathrm{CHCH}_{2}-\right)$. 
${ }^{29} \mathrm{SiNMR}\left(\mathrm{CDCl}_{3}, 600 \mathrm{MHz}\right): \delta(\mathrm{ppm}) 35.84$

Polymer, 3i

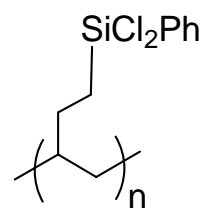

${ }^{1}$ HNMR $\left(\mathrm{CDCl}_{3}, 200 \mathrm{MHz}\right): \delta$ (ppm) 0.0-2.0 (br, 7H), 7.39 (m, 3H), $7.62(\mathrm{~m}, 2 \mathrm{H})$.

${ }^{13}$ CNMR/DEPT $\left(\mathrm{CDCl}_{3}, 200 \mathrm{MHz}\right): \delta$ (ppm) 16.58(- $\left.\mathrm{CH}_{2} \mathrm{CH}_{2} \mathrm{Si}-\right), 25.47\left(-\mathrm{CH}_{2} \mathrm{CH}_{2} \mathrm{Si}-\right)$, $33.18\left(-\mathrm{CHCH}_{2}-\right), 37.86\left(-\mathrm{CHCH}_{2}-\right), 128.34$ (-SiPh), 131.66 (-SiPh), 132.28 (-SiPh), $133.29(-\mathrm{SiPh})$.

${ }^{29} \mathrm{SiNMR}\left(\mathrm{CDCl}_{3}, 600 \mathrm{MHz}\right): \delta(\mathrm{ppm}) 20.24$.

Polymer, 3j

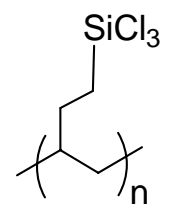

${ }^{1}$ HNMR (CDCl, $200 \mathrm{MHz}$ ): $\delta$ (ppm) 0.4-2.0 (br, 7H)

${ }^{13} \mathrm{CNMR} / \mathrm{DEPT}\left(\mathrm{CDCl}_{3}, 200 \mathrm{MHz}\right): \delta(\mathrm{ppm}) 20.2\left(-\mathrm{CH}_{2} \mathrm{CH}_{2} \mathrm{Si}-\right), 25.76\left(-\mathrm{CH}_{2} \mathrm{CH}_{2} \mathrm{Si}-\right)$, $32.97\left(-\mathrm{CHCH}_{2^{-}}\right), 37.83\left(-\mathrm{CHCH}_{2}-\right)$.

${ }^{29} \mathrm{SiNMR}\left(\mathrm{CDCl}_{3}, 600 \mathrm{MHz}\right): \delta(\mathrm{ppm}) 13.59$

Polymer, 3k

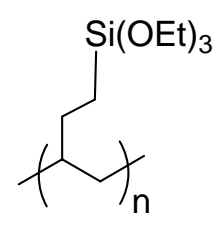

${ }^{1} \mathrm{HNMR}\left(\mathrm{CDCl}_{3}, 200 \mathrm{MHz}\right): \delta$ (ppm) 0.0-2.0 (br, 7H), 1.14 (t, 9H), 3.75 (q, 6H).

${ }^{13}$ CNMR/DEPT $\left(\mathrm{CDCl}_{3}, 200 \mathrm{MHz}\right): \delta(\mathrm{ppm}) 5.58\left(-\mathrm{CH}_{2} \mathrm{CH}_{2} \mathrm{Si}-\right), 17.93\left(-\mathrm{OCH}_{2} \mathrm{CH}_{3}\right)$, $25.39\left(-\mathrm{CH}_{2} \mathrm{CH}_{2} \mathrm{Si}-\right), 33.7\left(-\mathrm{CHCH}_{2^{-}}\right), 37.66\left(-\mathrm{CHCH}_{2}-\right), 57.85\left(-\mathrm{OCH}_{2} \mathrm{CH}_{3}\right)$.

${ }^{29} \mathrm{SiNMR}\left(\mathrm{CDCl}_{3}, 600 \mathrm{MHz}\right): \delta(\mathrm{ppm})-44.07$. 